\title{
Low-Loss and Low-Back-Reflection Hollow-Core to Standard Fiber Interconnection
}

\author{
M. Komanec, D. Suslov, S. Zvánovec, Y. Chen, T. Bradley, S. R. Sandoghchi, E. R. Numkam Fokoua, \\ G. T. Jasion, M. N. Petrovich, F. Poletti, D. J. Richardson, and R. Slavík
}

\begin{abstract}
We present a new approach to permanently interconnect hollow-core fiber (HCF) to solid-core fiber, which does not involve fusion splicing. Our approach is based on a modification of the glue-based fiber-array technology routinely used for fiber pigtailing of planar lightwave circuits. The resulting interconnection provides for a low insertion loss due to the fact that the HCF microstructure is not deformed during the gluing (low temperature) process that is almost impossible to achieve with the standard (high temperature) fusion splicing method. Furthermore, this low temperature technique enables the deposition and preservation of thin films deposited at the solid-to-hollow core fiber interface, allowing for additional functionality without the introduction of extra losses or any increase in complexity. To demonstrate this, we have applied an anti-reflection (AR) coating. A further feature of our approach is the ability to control very precisely the length of the graded-index (GRIN) fiber mode field (MF) adapter inserted in between the standard singlemode fiber (SMF-28) and the HCF. We show experimentally how the length of the GRIN fiber MF adapter influences the coupling between the SMF-28 and the fundamental as well as higher-order modes of the HCF. We coupled between SMF-28 $(10 \mu \mathrm{m}$ Mode Field Diameter, MFD) and the fundamental mode of a 19-cell hollow- core photonic bandgap fiber (HC-PBGF, 21.1 $\mu \mathrm{m}$ MFD) with the lowest-ever reported insertion loss of $0.30 \mathrm{~dB}$ per interface.
\end{abstract}

Index Terms-Optical components, Optical fiber connecting, Optical fibers

\section{INTRODUCTION}

$\mathbf{H}$ OLLOW-CORE fibers such as hollow-core photonic bandgap fibers (HC-PBGFs) offer many advantages over conventional solid-core fibers due to the strongly reduced interaction of the guided light with the glass material. Examples of the benefits of this are the extremely low fiber non-linearity, low and stable [1] signal latency, the possibility to construct long-length gas cells [2], etc.

A key property of any optical fiber is attenuation. In HC-PBGF the attenuation is defined principally by the fiber design. The common forms are the so-called 7-cell and 19-cell designs (made by removing 7 and 19 glass capillaries respectively to form the core in the preform from which the fiber is subsequently drawn). 7-cell HC-PBGFs typically have smaller cores (and mode field diameters, MFDs) and

M. Komanec, D. Suslov and S. Zvánovec are with the Faculty of Electrical Engineering, Czech Technical University in Prague, Prague, 16627, Czech Republic e-mail: komanmat@fel.cvut.cz

Y. Chen, T. Bradley, S. R. Sandoghchi, E. R. Numkam Fokoua, G. T. Jasion, M. N. Petrovich, F. Poletti, D. J. Richardson and R. Slavík are with the Optoelectronics Research Centre, University of Southampton, Southampton SO17 1BJ, United Kingdom

Manuscript received ....., 2018; revised ..., 2019. higher attenuation than 19-cell HC-PBGFs due to the increased interaction between the guided mode and the glass core surround (cladding). However, 7-cell HC-PBGF remains popular as it can be more straightforwardly interconnected to SMF-28 since both have a similar MFD. By contrast, when interconnecting 19-cell HC-PBGF [3] to SMF-28 there is a significant mismatch in MFD that needs to be managed e.g., using a mode field (MF) adapting bridge fiber.

Whilst generally operating in the single-mode regime both, 7-cell and 19-cell HC-PBGFs are inherently multi-moded, making the interconnection with SMF-28 prone to the unwanted coupling of part of the energy into higher-order modes (HOMs). This is highly undesirable in most applications, as it causes multi-path interference (MPI). To avoid this unwanted effect, the MFD and the MF profile of the fundamental modes of both interconnecting fibers must be very well matched; this is also needed to minimize the insertion loss (IL) of the interconnection.

There is sometimes an inconsistency in the literature when reporting insertion loss measurements for SMF-28-HC-PBGF interconnections. In some cases, the signal from the SMF-28 is coupled into the HC-PBGF and the power at the HC-PBGF output (total power in all of the excited guided modes) is measured. However, this does not represent the coupling loss between the fundamental mode of the SMF-28 and HC-PBGF. Moreover, due to multi-mode nature of HC-PBGFs, such measurements typically do not yield symmetric insertion loss values, i.e. the measured IL is different depending on whether the signal is propagating from the SMF-28 into the HC-PBGF, or vice-versa [4]. To properly characterize the coupling between the two fundamental fiber modes, it is important to measure only the power in the fundamental mode of HC-PBGF.

Apart from mitigating MPI and minimizing IL, the remaining challenges for hollow-core to solid-core fiber interconnection are the relatively strong Fresnel back-reflection and distortion of the HC-PBGF microstructure during the splicing process.

Regarding the solid-core to hollow-core interconnection IL, the best result reported up to date is $0.3 \mathrm{~dB}$ [5]. This result, however, was not achieved using standard SMF-28, but a custom-made large mode area single-mode fiber. The insertion loss was measured for a HC-PBGF interconnected to SMF-28 fiber at both ends. Both interconnections exhibited a loss of $0.3 \mathrm{~dB}$, confirming efficient coupling into the fundamental mode of the HC-PBGF. An anti-reflection (AR) coating was deposited on the large mode area fiber and this reduced the back-reflection to $-31 \mathrm{~dB}$ at $1550 \mathrm{~nm}$. The connection [5] 
was housed in a connector-style arrangement. This result is impressive and gives a benchmark as to how a coupling loss and back-reflection can be achieved with AR coatings. The main areas of improvements, however, are: (i) how to realize the low-loss connection with SMF-28, and (ii) how to hermetically seal the interconnection to avoid any degradation in the HCF loss with time (e.g., humidity-caused increase of attenuation).

Back-reflections can also be reduced by splicing angle-cleaved fibers to HC-PBGFs. The main drawback of this approach so far is in the relatively high level of IL $>3 \mathrm{~dB}$ [6] when 19-cell HC-PBGF was used (although an excellent $-60 \mathrm{~dB}$ level of back-reflection was achieved). With further refinement a reduced IL of 1-2 dB with a slight compromise in back-reflection $(-50 \mathrm{~dB})$ was reported [7]. The main challenges of this approach are the difficulty in reliably achieving accurate angle cleaves on such delicate structures and the high sensitivity of the IL to the cleave quality.

In this letter, we demonstrate a novel approach for low-loss, low back-reflection and highly-efficient fundamental-mode coupling between SMF-28 fiber and HC-PBGF. Our approach is based on fiber-array (FA) interconnection technology [8] which can be used to create a robust and permanent HC-PBGF to SMF-28 interconnection, whilst at the same time hermetically sealing the end-facet of the HC-PBGF.

We incorporate a graded-index (GRIN) fiber based MF adapter and demonstrate how sensitive low-loss coupling between the SMF-28 and the fundamental mode of the HC-PBGF is to the precise length of the GRIN fiber used. We achieved ILs as low as $0.30 \mathrm{~dB}$ between the fundamental modes of the two fibers (a record value); with a reduced back-reflection loss of $-30 \mathrm{~dB}$. We also characterized the level of MPI and measured the polarization-dependent loss.

\section{MODIFIED FIBER-ARRAY TECHNOLOGY}

Our approach is based on the FA assembly technique, which is a well-established method to pigtail photonic lightwave circuits (PLCs) with solid-core optical fibers. The fibers are first glued into a V-groove array, which is then glued onto the PLC end-facet. Adaptation is required to apply this basic approach to the case of HC-PBGF to solid-core fiber interconnection and a schematic of the modified FA assembly is depicted in Fig. 1 along with the principle of MF adaptation using a GRIN fiber segment.

First we use a 1-channel FA for the solid-core fiber and MF adaptation, we fusion splice GRIN fiber to the end of the input SMF-28. This MF adaptation technique has previously been used to make connectors for high power laser applications [9] in which a large MFD (and thus lower power density) is required at the point of interconnection. We cleave the GRIN fiber to a length longer than the target length and insert the spliced SMF-28 and GRIN segment into the V-groove before gluing it in place. Finally, we polish the fiber-array end-face until the desired GRIN length is achieved. This process allows for very precise control of the GRIN fiber length ( $\mu \mathrm{m}$-level), which is essential for low-loss interconnection, as we shall show later. Subsequently, for this proof-of-principle demonstration, we deposit a simple $\mathrm{TiO}_{2} / \mathrm{SiO}_{2}$ 2-layer AR coating
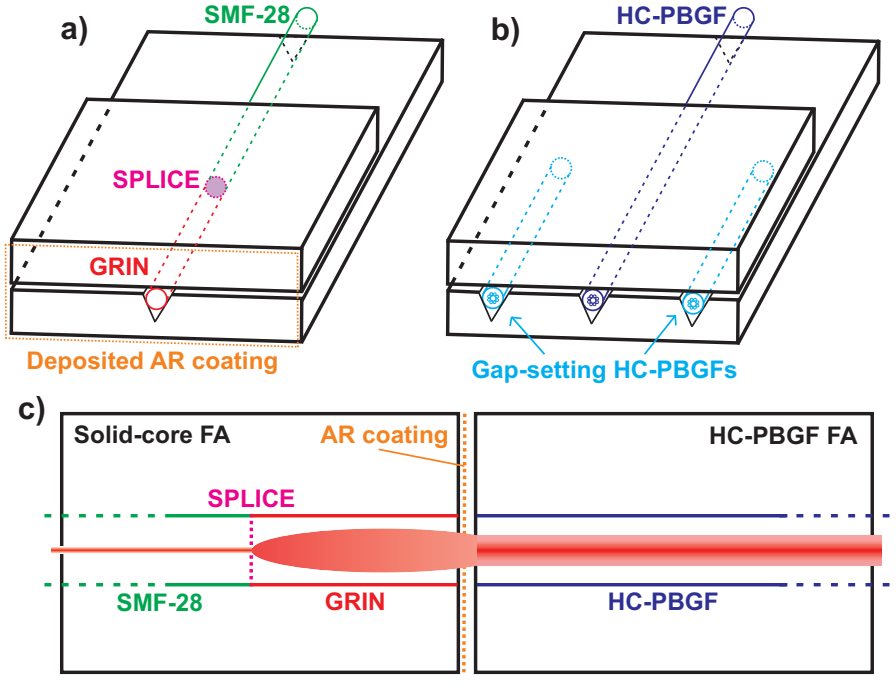

Fig. 1. Illustration of the developed 1-channel FA, a) containing SMF28 spliced on GRIN, with the GRIN length precisely polished and with a subsequently deposited AR coating, b) containing HC-PBGF inserted and aligned with the FA end-facet. Two short segments of the same-diameter HCPBGF are used to set the vertical gap size, c) with a schematic of the MF propagation and MF matching at the GRIN-HC-PBGF interface.

on the GRIN end-facet (see the SMF-28-GRIN component in Fig. 1a). The AR coating had the minimum reflection of $-32 \mathrm{~dB}$ at $1600 \mathrm{~nm}$ and a 3-dB bandwidth of $60 \mathrm{~nm}$.

Simulations (ray-tracing in ZEMAX) indicated that the best commercially available GRIN fiber has a core diameter of $62.5 \mu \mathrm{m}$ (standard OM1 graded-index multi-mode fiber). For a fully-collimating (quarter-pitch, length $265 \mu \mathrm{m}$ ) GRIN, it gives MFD of $24.6 \mu \mathrm{m}$, which is close to the MFD of the fundamental mode in HC-PBGF.

HC-PBGF cannot be polished in the V-groove in the same way as the solid-core GRIN fiber because of fear of damage to the delicate microstructure and ingression of polishing products. Thus, we modified the FA assembly procedure for HC-PBGF, which also takes into account the fact that HC-PBGFs generally have non-standard outer diameters which can vary from HC-PBGF sample to sample. The FA is a 1-channel type, but includes three V-grooves. The two side V-grooves are used to set the vertical gap between the upper and lower blocks of the FA by using HC-PBGF segments made of the same fiber (same diameter) as that to be used in the interconnection. At this stage, we intentionally leave the central V-groove empty. Subsequently, the HC-PBGF FA is polished and cleaned. After that, we insert a freshly cleaved $\mathrm{HC}$-PBGF into the central $\mathrm{V}$-groove and precisely align its end-face with the front edge of the FA (see the HC-PBGF component in Fig. 1b).

\section{MeAsurement SETUP}

The HC-PBGF used in our experiments was an in-house made 19-cell HC-PBGF with a $230 \mu \mathrm{m}$ outer diameter and a $31.4 \mu \mathrm{m}$ central air-hole diameter. The MFD of the fundamental mode was calculated (in COMSOL) to be $21.1 \mu \mathrm{m}$ at $1550 \mathrm{~nm}$. Calculated interconnection loss between the calculated shape of the HC-PBGF mode and a Gaussian beam 
of $21.1 \mu \mathrm{m}$ (ideal) and $24.6 \mu \mathrm{m}$ (our quarter-pitch GRIN lens) was $0.13 \mathrm{~dB}$ and $0.23 \mathrm{~dB}$, respectively. We prepared two samples, having $10 \mathrm{~m}$ and $50 \mathrm{~m}$ HC-PBGF lengths, and made interconnections to SMF-28 fiber pigtails at each side.

For precise alignment, the FAs were placed on 5-axis micropositioning stages (X,Y,Z, pitch, and yaw), as shown in Fig. 2. We used a stabilized Erbium-doped fiber amplifier (EDFA) as a broadband light source. The EDFA output (ASE) was coupled into an optical bandpass filter (OBPF) with $10 \mathrm{~nm}$ bandwidth. The light then passed through a circulator (CIRC), a fiber polarization controller (PC), and the first SMF28-GRIN-HC-PBGF. After the HC-PBGF sample, the signal was coupled through the second HC-PBGF-GRIN-SMF-28 (again, using 5-axis micropositioning stage) and was measured with the optical spectrum analyzer (OSA) or power meter (PM). The spectrum of the reflected signal was measured at the 3rd CIRC port.

a)

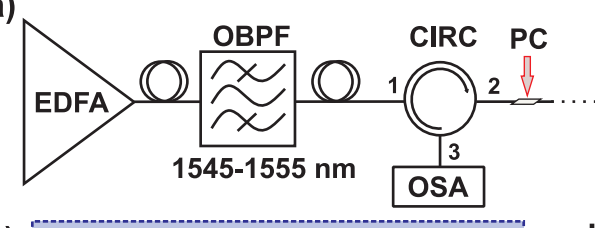

b)

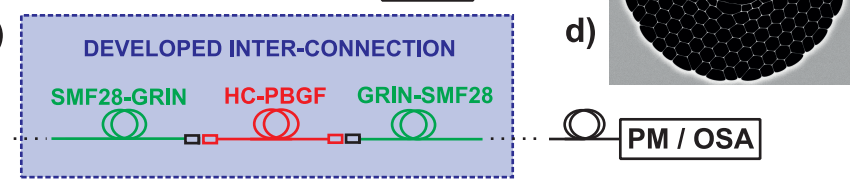

c)

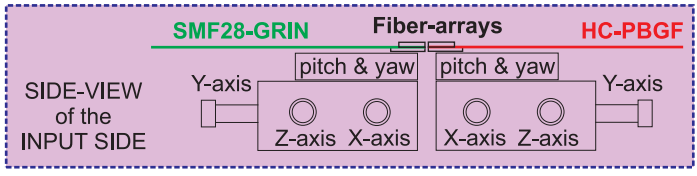

Fig. 2. Measurement configuration including a) input signal generation, b) developed interconnection with output power/spectral measurement, c) sideview of the alignment apparatus and d) 19-cell HC-PBGF structure. EDFA - Erbium-doped fiber amplifier, OBPF - optical bandpass filter, CIRC circulator, PC - polarization controller, GRIN - graded-index fiber MF adapter, PM - power meter, OSA - optical spectrum analyzer.

\section{REsults}

First, we measured the IL dependence on the GRIN length. Our preliminary experimental results (not shown here) indicated that the optimum GRIN length is slightly longer than that expected from the theoretical analysis. We used five pairs of GRIN-SMF-28 FA with the 2-layer ARC and GRIN lengths (the same lengths in each pair) of 290, 295, 300,305 and $315 \mu \mathrm{m}$, respectively. The GRIN lengths were measured using an optical microscope, measuring the splice to end-facet distance with a measurement uncertainty of $\pm 1 \mu \mathrm{m}$. The measured IL for the whole SMF-28-GRINHC-PBGF-GRIN-SMF-28 assembly is plotted in Fig 3.

The minimum IL was obtained with a GRIN length of $295 \mu \mathrm{m}$. It is worth noting that deviation as little as $5 \mu \mathrm{m}$ from the optimum GRIN length leads to the insertion loss increase of $>0.1-0.2 \mathrm{~dB}$ (see Fig. 3). A similar level of insertion loss increase is expected (based on a numerical analysis) in the case of longitudinal or transverse misalignment of $\sim 5 \mu \mathrm{m}$. Note that

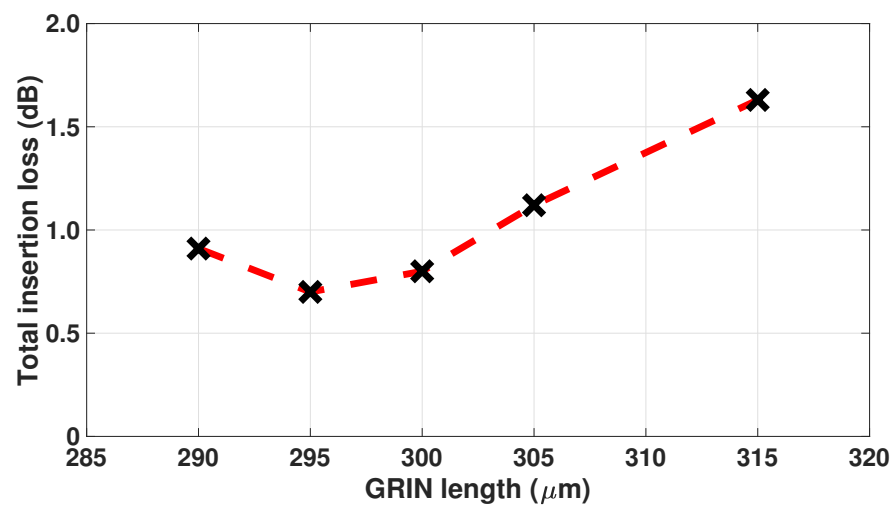

Fig. 3. Dependence of total insertion loss of the whole SMF-GRIN-HCPBGF-GRIN-SMF component on the GRIN fiber MF adapter length (the same GRIN length was always used on both sides), utilizing a 10-m long 19-cell HC-PBGF.

besides slightly increased insertion loss, any misalignment will result as well in stronger unwanted HOM excitation.

Besides optimizing the IL using the two 5-axes stages, we also monitored the quality of the launch into the fundamental mode by observing the transmitted signal with the OSA. Any component of power launched into HOMs presents itself as MPI and is visible as spectral ripples on the transmitted broadband spectrum measured at the OSA. This technique is simpler than conventional measurements of MPI based on the time-of-flight technique [10] or $S^{2}$ measurement [10], [11]. The HOMs contributing most to MPI are the $\mathrm{LP}_{11}$ mode and the $\mathrm{LP}_{02}$ mode with differential modal delays of $8.7 \mathrm{ps} / \mathrm{m}$ and $21.4 \mathrm{ps} / \mathrm{m}$ respectively [11]. We should be able to observe MPI from these modes in HC-PBGF with lengths up to $45 \mathrm{~m}$ and $18 \mathrm{~m}$ length respectively when operating the OSA at its highest resolution of $20 \mathrm{pm}$.

The MPI for various GRIN lengths used for the 10-m long HC-PBGF sample is shown in Fig. 4, together with the measured IL for the entire SMF28-GRIN-HC-PBGF-GRIN-SMF-28 assembly.

The minimum MPI (demonstrated as the smallest amplitude spectral ripples) was achieved for a GRIN fiber length of 295 to $300 \mu \mathrm{m}$ (the minimum IL was achieved for the $295 \mu \mathrm{m}$ length).

Once we optimized the optical performance, we glued the FAs together. Apart from ensuring that the connection does not move during the glue-curing process, we had also to ensure that the glue does not creep into the HC-PBGF microstructured region - we used a fast $(<5 \mathrm{~s})$ UV-curable, high viscosity glue for this purpose. First, we aligned both 5-axis stages for the entire SMF-28-GRIN-HC-PBGF-GRIN-SMF-28 assembly. During the gluing, (the first interconnection followed by the second one), we observed a maximum increase in the IL per interface of $0.05 \mathrm{~dB}$.

Subsequently, we interconnected a 50-m long HC-PBGF. We achieved an IL of $0.87 \pm 0.05 \mathrm{~dB}$ (which also includes the PDL) for the complete SMF-28-GRIN-HC-PBGF-GRIN-SMF-28 glued assembly. Considering the loss of the 50-m long HC-PBGF $(4.0 \mathrm{~dB} / \mathrm{km})$, we calculated IL per connection of $0.30 \mathrm{~dB}$. Figure 6 shows 


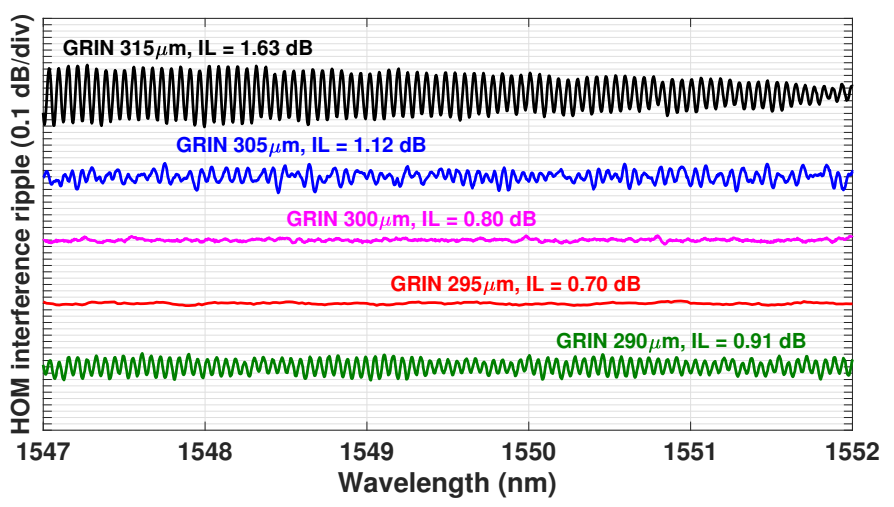

Fig. 4. HOM interference ripples in transmitted spectra for various GRIN fiber MF adapter lengths measured for the whole SMF28-GRIN-HC-PBGFGRIN-SMF28 configuration including a 10-m long HC-PBGF.

the measured back-reflection spectrum of the 50-m long HC-PBGF after the assembly process (without the OBPF). We observed $15 \mathrm{~dB}$ lower back-reflection at $1580 \mathrm{~nm}$ (the used ARC showed minimum reflection there) with respect to the measured $4 \%$ reflection from a flat interface.

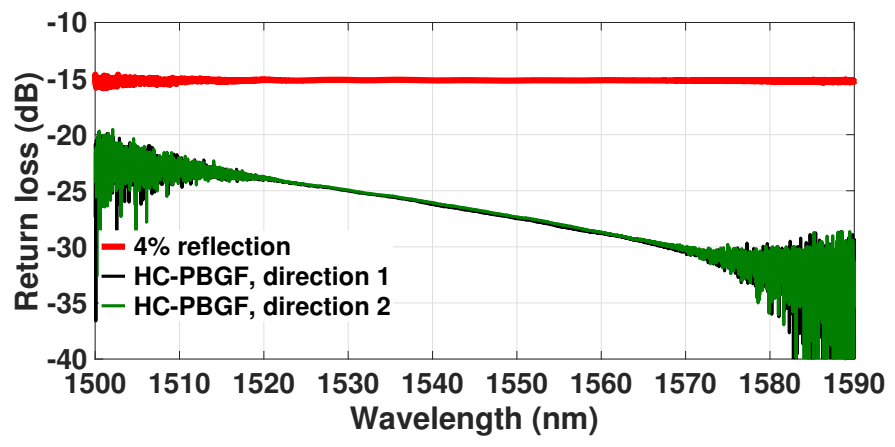

Fig. 5. Back-reflection spectra of the final component including 50-m long HC-PBGF.

\section{CONCLUSiON}

In this paper we have presented for the first time a FA based approach to create a permanent hermetically sealed interconnection of HC-PBGF to SMF-28s. Our method provides signal propagation in the fundamental mode, with the signal propagating in the higher-order modes being significantly suppressed, reducing multipath propagation effects.

Using our approach, a coating can be applied to the interface. As a proof-of principle, we have reduced back-reflections by $15 \mathrm{~dB}$ compared to the typical silica-air interface by depositing a simple 2-layer AR coating. Apart from reducing the back-reflection, the AR coating is also expected to reduce IL by $0.17 \mathrm{~dB}$ per silica-air interface.

We reached best-ever reported insertion loss between SMF-28 and 19-cell HC-PBGF of $0.30 \mathrm{~dB}$, confirmed both for a 10-m long and 50-m long HC-PBGF. The interconnection was reciprocal, with IL difference of only $0.03 \mathrm{~dB}$ for both propagation directions, confirming good coupling between fundamental modes of both fibers. Furthermore we have pre- sented a simple approach of single-mode launch verification by monitoring MPI with a spectrum analyzer.

An additional advantage of the presented method lies is the fact that it can be straightforwardly extended to simultaneously interconnecting several (e.g., 64) HC-PBGFs with multiple SMF-28s or even with a multi-port planar lightwave circuit (e.g., a 64x64 PLC coupler).

\section{ACKNOWLEDGMENT}

This research was supported by the following grants: CTU in Prague: (SGS17/182/OHK3/3T/13); ORC: UK RAEng (Senior Research Fellowship - RCSFR1718 \6\15); Engineering and Physical Sciences Research Council (EPSRC) (EP/P030181/1). The data in this paper is accessible through the University of Southampton research repository (DOI: 10.5258/SOTON/D0831).

\section{REFERENCES}

[1] R. Slavík, G. Marra, E. N. Fokoua, N. Baddela, N. V. Wheeler, M. Petrovich, F. Poletti, and D. J. Richardson, "Ultralow thermal sensitivity of phase and propagation delay in hollow core optical fibres," Scientific reports, vol. 5, OCT 222015.

[2] N. Dadashzadeh, M. P. Thirugnanasambandam, H. W. K. Weerasinghe, B. Debord, M. Chafer, F. Gerome, F. Benabid, B. R. Washburn, and K. L. Corwin, "Near diffraction-limited performance of an OPA pumped acetylene-filled hollow-core fiber laser in the mid-IR," Opt. Express, vol. 25, no. 12, pp. 13351-13358, Jun 2017.

[3] Y. Chen, Z. Liu, S. R. Sandoghchi, G. T. Jasion, T. D. Bradley, E. N. Fokoua, J. R. Hayes, N. V. Wheeler, D. R. Gray, B. J. Mangan, R. Slavik, F. Poletti, M. N. Petrovich, and D. J. Richardson, "Multi-kilometer long, longitudinally uniform hollow core photonic bandgap fibers for broadband low latency data transmission," Journal of Lightwave Technology, vol. 34, no. 1, pp. 104-113, Jan 2016.

[4] R. Thapa, K. Knabe, K. L. Corwin, and B. R. Washburn, "Arc fusion splicing of hollow-core photonic bandgap fibers for gas-filled fiber cells," Opt. Express, vol. 14, no. 21, pp. 9576-9583, Oct 2006.

[5] J. W. Nicholson, B. Mangan, L. Meng, A. DeSantolo, R. S. Windeler, J. M. Fini, K. Mukasa, E. M. Monberg, P. Vannasouk, E. Warych, and T. Taunay, "Low-loss, low return-loss coupling between SMF and single-mode, hollow-core fibers using connectors," in 2014 Conference on Lasers and Electro-Optics (CLEO) - Laser Science to Photonic Applications, June 2014, pp. 1-2.

[6] F. Couny, F. Benabid, and P. S. Light, "Reduction of Fresnel BackReflection at Splice Interface Between Hollow Core PCF and SingleMode Fiber," IEEE Photonics Technology Letters, vol. 19, no. 13, pp. $1020-1022$, July 2007.

[7] G. A. Miller and G. A. Cranch, "Reduction of Intensity Noise in Hollow Core Optical Fiber Using Angle-Cleaved Splices," IEEE Photonics Technology Letters, vol. 28, no. 4, pp. 414-417, Feb 2016.

[8] N. Hiroi, Y. Hashimoto, and K. Matsumoto, "Optical fiber array," Patent, May 3, 2011, US Patent US20090285533A1.

[9] Y. Shou and J. Zhao, "Mode field expanded fiber collimator," Patent, 5, 2011, US Patent US7920763B1.

[10] D. R. Gray, Z. Li, F. Poletti, R. Slavík, N. V. Wheeler, N. K. Baddela, M. N. Petrovich, A. Obeysekara, and D. J. Richardson, "Complementary analysis of modal content and properties in a 19-cell hollow core photonic band gap fiber using time-of-flight and $\mathrm{S}^{2}$ techniques," in 2012 38th European Conference and Exhibition on Optical Communications, Sept 2012, pp. 1-4.

[11] D. R. Gray, S. R. Sandoghchi, N. V. Wheeler, N. K. Baddela, G. T. Jasion, M. N. Petrovich, F. Poletti, and D. J. Richardson, "Accurate calibration of $S^{2}$ and interferometry based multimode fiber characterization techniques," Opt. Express, vol. 23, no. 8, pp. 10 540-10 552, Apr 2015. 